\title{
On a smooth compactification of $\operatorname{PSL}(n, \mathbb{C}) / T$
}

\author{
Indranil Biswas, S. Senthamarai Kannan, and D. S. Nagaraj
}

\begin{abstract}
Let $T$ be a maximal torus of $\operatorname{PSL}(n, \mathbb{C})$. For $n \geq 4$, we construct a smooth compactification of $\operatorname{PSL}(n, \mathbb{C}) / T$ as a geometric invariant theoretic quotient of the wonderful compactification $\overline{\operatorname{PSL}(n, \mathbb{C})}$ for a suitable choice of $T$-linearized ample line bundle on $\overline{\operatorname{PSL}(n, \mathbb{C})}$. We also prove that the connected component, containing the identity element, of the automorphism group of this compactification of $\operatorname{PSL}(n, \mathbb{C}) / T$ is $\operatorname{PSL}(n, \mathbb{C})$ itself.
\end{abstract}

\section{Introduction}

Let $G$ be a semisimple group of adjoint type over the field $\mathbb{C}$ of complex numbers. De Concini and Procesi [DP] constructed a smooth projective variety $\bar{G}$ with an action of $G \times G$ such that

- the variety $G$ equipped with the action of $G \times G$ given by the left and right translations is an open dense orbit of it, and

- the boundary $\bar{G} \backslash G$ is a union of $G \times G$ stable normal crossing divisors.

This variety $\bar{G}$ is known as the wonderful compactification of $G$.

Fix a maximal torus $T$ of $G$. Consider the right action of $T$ on $\bar{G}$, meaning the action of the subgroup $1 \times T \subset G \times G$. For a $T$-linearized ample line bundle $\mathcal{L}$ on $\bar{G}$, let $\bar{G}_{T}^{s s}(\mathcal{L})$ and $\bar{G}_{T}^{s}(\mathcal{L})$ denote, respectively, the loci of semistable and stable points of $\bar{G}$ (see [MFK, p. 30, p. 40]).

Our first main result (Proposition 3.3) says that there is a $T$-linearized ample line bundle $\mathcal{L}$ on $\bar{G}$ such that $\bar{G}_{T}^{s s}(\mathcal{L})=\bar{G}_{T}^{s}(\mathcal{L})$. For $G=\operatorname{PSL}(n, \mathbb{C})$, we show that there is a $T$-linearized ample line bundle $\mathcal{L}$ on $\overline{\operatorname{PSL}(n, \mathbb{C})}$ such that

- the geometric invariant theoretic (GIT) quotient $\overline{\operatorname{PSL}(n, \mathbb{C})_{T}^{s s}}(\mathcal{L}) / / T$ is smooth, and

- the boundary $\left(\overline{\operatorname{PSL}(n, \mathbb{C})}_{T}^{s s}(\mathcal{L}) / / T\right) \backslash(\operatorname{PSL}(n, \mathbb{C}) / T)$ is a union of $\operatorname{PSL}(n, \mathbb{C})$ stable normal crossing divisors.

Kyoto Journal of Mathematics, Vol. 56, No. 1 (2016), 165-175

DOI 10.1215/21562261-3445183, (C) 2016 by Kyoto University

Received August 26, 2012. Revised January 16, 2015. Accepted January 16, 2015.

2010 Mathematics Subject Classification: 14F17.

Biswas's work supported by a J. C. Bose Fellowship.

Kannan's work supported in part by the Infosys Foundation. 
We further show that, for $n \geq 4$, the connected component of the automorphism group of $\overline{\operatorname{PSL}(n, \mathbb{C})_{T}^{s s}} / / T$ containing the identity automorphism is $\operatorname{PSL}(n, \mathbb{C})$ (Theorem 4.1).

\section{Preliminaries and notation}

In this section we recall some preliminaries and notation about Lie algebras and algebraic groups (see, e.g., [Hu1] and [Hu2] for the details). Let $G$ be a simple group of adjoint type of rank $n$ over the field of complex numbers. Let $T$ be a maximal torus of $G$, and let $B \supset T$ be a Borel subgroup of $G$. Let $N_{G}(T)$ denote the normalizer of $T$ in $G$. So $W:=N_{G}(T) / T$ is the Weyl group of $G$ with respect to $T$.

The Lie algebra of $G$ will be denoted by $\mathfrak{g}$. Let $\mathfrak{h} \subset \mathfrak{g}$ be the Lie algebra of $T$. The set of roots of $G$ with respect to $T$ will be denoted by $R$. Let $R^{+} \subset R$ be the set of positive roots with respect to $B$. Let

$$
S=\left\{\alpha_{1}, \alpha_{2}, \ldots, \alpha_{n}\right\} \subset R^{+}
$$

be the set of simple roots with respect to $B$. The group of characters of $T$ will be denoted by $X(T)$, while the group of one-parameter subgroups of $T$ will be denoted by $Y(T)$. Let

$$
\left\{\lambda_{i} \mid 1 \leq i \leq n\right\}
$$

be the ordered set of one-parameter subgroups of $T$ satisfying the condition that $\left\langle\alpha_{i}, \lambda_{j}\right\rangle=\delta_{i j}$, where

$$
\langle\cdot, \cdot\rangle: X(T) \times Y(T) \longrightarrow \mathbb{Z}
$$

is the natural pairing, and $\delta_{i j}$ is the Kronecker delta function. Let $\leq$ (resp., $\geq$ ) be the partial order on $X(T)$ defined as follows: $\chi_{1} \leq \chi_{2}$ (resp., $\chi_{1} \geq \chi_{2}$ ) if $\chi_{2}-\chi_{1}$ (resp., $\chi_{1}-\chi_{2}$ ) is a linear combination of simple roots with nonnegative integers as coefficients.

Let $(\cdot, \cdot)$ denote the restriction of the Killing form of $\mathfrak{g}$ to $\mathfrak{h}$. Let

$$
\left\{\omega_{j} \mid 1 \leq j \leq n\right\}
$$

be the ordered set of fundamental weights corresponding to $S$; in other words,

$$
\frac{2\left(\omega_{i}, \alpha_{j}\right)}{\left(\alpha_{j}, \alpha_{j}\right)}=\delta_{i j}, \quad 1 \leq i, j \leq n .
$$

For $1 \leq i \leq n$, let $s_{\alpha_{i}}$ denote the simple reflection corresponding to $\alpha_{i}$.

The longest element of $W$ corresponding to $B$ will be denoted by $w_{0}$. Let

$$
B^{-}=w_{0} B w_{0}^{-1}
$$

be the Borel subgroup of $G$ opposite to $B$ with respect to $T$. For the notion of a $G$-linearization, and the GIT quotients, we refer to [MFK, p. 30, p. 40].

Consider the flag variety $G / B$ that parameterizes all Borel subgroups of $G$. For a character $\chi$ of $B$, let

$$
L_{\chi}=G \times{ }_{B} \mathbb{C} \longrightarrow G / B
$$


be the $G$-linearized line bundle associated to the action of $B$ on $G \times \mathbb{C}$ given by $b .(g, z)=\left(g b, \chi\left(b^{-1}\right) z\right)$ for $b \in B$ and $(g, z) \in G \times \mathbb{C}$. So, in particular, $L_{\chi}$ is $T$-linearized. When $L_{\chi}$ is ample, we denote by $(G / B)_{T}^{s s}\left(L_{\chi}\right)\left(\operatorname{resp} .,(G / B)_{T}^{s}\left(L_{\chi}\right)\right)$ the semistable (resp., stable) locus in $G / B$ for the $T$-linearized ample line bundle $L_{\chi}$.

Next we recall some facts about the wonderful compactification of $G$. Let $\chi$ be a regular dominant weight of $G$ with respect to $T$ and $B$, and let $V(\chi)$ be the irreducible representation of $\widehat{G}$ with highest weight $\chi$, where $\widehat{G}$ is the simply connected covering of $G$. By [DP, p. 16, Section 3.4], the wonderful compactification $\bar{G}$, which we denote by $X$, is the closure of the $(G \times G)$-orbit of the point

$$
[1] \in \mathbb{P}\left(V(\chi) \otimes V(\chi)^{*}\right)
$$

corresponding to the identity element 1 of $V(\chi) \otimes V(\chi)^{*}=\operatorname{End}\left(V(\chi)^{*}\right)$. We denote by $\mathcal{L}_{\chi}$ the ample line bundle on $X$ induced by this projective embedding. Since the regular dominant weights generate the weight lattice, given a weight $\chi$, we have the line bundle $\mathcal{L}_{\chi}$ on $X$ associated to $\chi$.

By [DP, Theorem, p. 14, Section 3.1], there is a unique closed $(G \times G)$-orbit $Z$ in $X$. Note that

$$
Z=\bigcap_{i=1}^{n} D_{i},
$$

where $D_{i}$ is the $G \times G$ stable irreducible component of $\bar{G} \backslash G$ such that $\mathcal{O}\left(D_{i}\right)=$ $\mathcal{L}_{\alpha_{i}}$ (see [DP, p. 29, Section 8.2, Corollary]). Further, $Z$ is isomorphic to $G / B \times$ $G / B^{-}$as a $G \times G$ variety. By [DP, p. 26, Section 8.1], the pullback homomorphism

$$
i^{*}: \operatorname{Pic}(X) \longrightarrow \operatorname{Pic}(Z)
$$

for the inclusion map $i: Z \hookrightarrow X$ is injective and is given by

$$
i^{*}\left(\mathcal{L}_{\chi}\right)=p_{1}^{*}\left(L_{\chi}\right) \otimes p_{2}^{*}\left(L_{-\chi}\right),
$$

where $L_{\chi}$ (resp., $L_{-\chi}$ ) is the line bundle on $G / B$ (resp., $G / B^{-}$) associated to $\chi$ (resp., $-\chi$ ), and $p_{j}$ is the projection to the $j$ th factor of $G / B \times G / B^{-}$for $j=1,2$.

\section{Choice of a polarization on $\bar{G}$}

We continue with the notation of Section 2. Let $G$ be a simple algebraic group of adjoint type of rank $n \geq 2$ such that its root system $R$ is different from $A_{2}$. Let

$$
\mathbb{N} S:=\left\{\sum_{i=1}^{n} m_{i} \alpha_{i}: m_{i} \in \mathbb{N}\right\} .
$$

Then, we have the following.

LEMMA 3.1

The above defined $\mathbb{N} S$ contains a regular dominant character $\chi$ of $T$ such that $s_{\alpha_{i}}(\chi) \geq 0$ and $\left\langle\chi, w\left(\lambda_{i}\right)\right\rangle \neq 0$ for every $w \in W$ and $1 \leq i \leq n$. 
Proof

Denote by $X(T)_{\mathbb{Q}}$ the rational vector space generated by $X(T)$, and also denote by $X(T)^{+}$the semigroup of it given by the dominant characters of $T$. Let $\rho \in$ $X(T)_{\mathbb{Q}}$ be the half-sum of positive roots of $R$. Then, $2 \rho=2\left(\sum_{i=1}^{n} \omega_{i}\right) \in X(T)^{+}$ is a regular dominant character of $T$, and we have $2 \rho \in \mathbb{N} S$.

Since $R$ is irreducible of rank at least 2 and different from $A_{2}$, we see that, for every simple root $\alpha_{i}$, there are at least three positive roots $\beta$ satisfying $\alpha_{i} \leq \beta$. Hence, the coefficient of every simple root $\alpha_{j}$ in the expression of $s_{\alpha_{i}}(2 \rho)=$ $2 \rho-2 \alpha_{i}$ (as a nonnegative integral linear combination of simple roots) is positive. Hence, we have $s_{\alpha_{i}}(2 \rho) \in \mathbb{N} S$. Thus, we have

$$
2 \rho \in X(T)^{+} \cap\left(\bigcap_{i=1}^{n} s_{\alpha_{i}}(\mathbb{N} S)\right) .
$$

Denote by $N$ the determinant of the Cartan matrix of $R$. Then we have $N \omega_{i} \in \mathbb{N} S$ for every $i=1,2, \ldots, n$. By the previous discussion, there exists $m \in \mathbb{N}$ such that $m s_{\alpha_{i}}(2 \rho)-N \alpha_{i} \in \mathbb{N} S$ for every $1 \leq i \leq n$. Hence, we get

$$
s_{\alpha_{i}}\left(2 m \rho+N \omega_{i}\right)=m s_{\alpha_{i}}(2 \rho)-N \alpha_{i}+N \omega_{i} \in \mathbb{N} S, \quad 1 \leq i \leq n,
$$

and from this it follows that

$$
2 m \rho+N \omega_{i} \in X(T)^{+} \cap\left(\bigcap_{j=1}^{n} s_{\alpha_{j}}(\mathbb{N} S)\right), \quad 1 \leq i \leq n .
$$

Consider the characters $2 m \rho, 2 m \rho+N \omega_{2}, \ldots, 2 m \rho+N \omega_{n}$ of $T$. These are linearly independent in $X(T)$ and by construction they all lie in the rational cone

$$
\mathcal{C} \subset X(T)_{\mathbb{Q}}^{+}
$$

generated by the semigroup $X(T)^{+} \cap\left(\bigcap_{i=1}^{n} s_{\alpha_{i}}(\mathbb{N} S)\right)$. It follows that $\mathcal{C}$ has a maximal dimension in $X(T)_{\mathbb{Q}}$; hence it is not contained in any hyperplane of $X(T)_{\mathbb{Q}}$. Therefore, there exists a regular dominant character $\chi \in \mathcal{C} \cap \mathbb{N} S$ such that $\left\langle\chi, w\left(\lambda_{i}\right)\right\rangle \neq 0$ for all $1 \leq i \leq n$ and every $w \in W$, and hence the lemma follows.

\section{LEMMA 3.2}

Let $\chi \in \mathbb{N} S$ be a regular dominant character of $T$ satisfying the properties stated in Lemma 3.1. Then we have

(a) $(G / B)_{T}^{s s}\left(L_{\chi}\right)=(G / B)_{T}^{s}\left(L_{\chi}\right)$, and

(b) the set of all unstable points

$$
(G / B) \backslash(G / B)_{T}^{s s}\left(L_{\chi}\right)
$$

is contained in the union of $W$-translates of all Schubert varieties of codimension at least two. 
Proof

Set $L:=L_{\chi}$. Since $\left\langle\chi, w\left(\lambda_{i}\right)\right\rangle \neq 0$ for every $w \in W$ and $1 \leq i \leq n$, by [Ka1, p. 38, Lemma 4.1] we have

$$
(G / B)_{T}^{s s}(L)=(G / B)_{T}^{s}(L)
$$

This proves (a).

To prove (b), take an unstable point $x \in G / B$ for the polarization $L$. Then, there is a one-parameter subgroup $\lambda$ of $T$ such that $\mu^{L}(x, \lambda)<0$. Let $\phi \in W$ be such that $\phi(\lambda)$ is in the fundamental chamber, say,

$$
\phi(\lambda)=\sum_{i=1}^{n} c_{i} \lambda_{i}
$$

where $\left\{c_{i}\right\}$ are nonnegative integers. Consequently, we have

$$
\mu^{L}\left(n_{\phi}(x), \phi(\lambda)\right)=\mu^{L}(x, \lambda)<0,
$$

where $n_{\phi}$ is a representative of $\phi$ in $N_{G}(T)$. Now, let $n_{\phi}(x)$ be in the Schubert cell $B w B / B$ for some $w \in W$. By [Se, Lemma 5.1], we have

$$
\mu^{L}\left(n_{\phi}(x), \phi(\lambda)\right)=\left(-\sum_{i=1}^{n} c_{i}\left\langle w(\chi), \lambda_{i}\right\rangle\right)<0 .
$$

(The sign here is negative because we are using the left action of $B$ on $G / B$ while in [Se, Lemma 5.1] the action of $B$ on $B \backslash G$ is on the right.) Therefore we have $w(\chi) \not \leq 0$. For every $1 \leq i \leq n$ we have $s_{\alpha_{i}}(\chi) \geq 0$, and hence $w_{0} s_{\alpha_{i}}(\chi) \leq 0$. Hence we have $l\left(w_{0}\right)-l(w) \geq 2$. This completes the proof of (b).

\section{PROPOSITION 3.3}

Let $X=\bar{G}$ be the wonderful compactification of $G$. Let $\chi$ be as in Lemma 3.2, and let $X_{T}^{s s}\left(\mathcal{L}_{\chi}\right)$ (resp., $X_{T}^{s}\left(\mathcal{L}_{\chi}\right)$ ) be the semistable (resp., stable) locus of $X$ for the action of $1 \times T$ and the polarization $\mathcal{L}_{\chi}$ on $X$. Then we have

(1) $X_{T}^{s s}\left(\mathcal{L}_{\chi}\right)=X_{T}^{s}\left(\mathcal{L}_{\chi}\right)$, and

(2) the set of unstable points $X \backslash\left(X_{T}^{s s}\left(\mathcal{L}_{\chi}\right)\right)$ is a union of irreducible closed subvarieties of codimension at least three.

\section{Proof}

Let $Z$ be the unique closed $(G \times G)$-orbit in $X$. Let $Z_{T}^{s s}\left(\mathcal{L}_{\chi}\right)\left(\operatorname{resp} ., Z_{T}^{s}\left(\mathcal{L}_{\chi}\right)\right)$ be the semistable (resp., stable) locus of $Z$ for the action of $1 \times T$ and the polarization $i^{*}\left(\mathcal{L}_{\chi}\right)$, where $i: Z \hookrightarrow X$ is the inclusion map. Since $Z$ is isomorphic to $G / B \times G / B^{-}$and $i^{*}\left(\mathcal{L}_{\chi}\right)=p_{1}^{*}\left(L_{\chi}\right) \otimes p_{2}^{*}\left(L_{-\chi}\right)$, we see that

$$
Z_{T}^{s s}\left(\mathcal{L}_{\chi}\right) \simeq(G / B) \times\left(\left(G / B^{-}\right)_{T}^{s s}\left(L_{-\chi}\right)\right)
$$

and $Z_{T}^{s}\left(\mathcal{L}_{\chi}\right) \simeq(G / B) \times\left(\left(G / B^{-}\right)_{T}^{s}\left(L_{-\chi}\right)\right)$. Set $Z^{s s}=Z_{T}^{s s}\left(\mathcal{L}_{\chi}\right)$, and set $Z^{s}=$ $Z_{T}^{s}\left(\mathcal{L}_{\chi}\right)$. By Lemma 3.2 and the above discussion, we have $Z^{s s}=Z^{s}$.

For convenience, we will denote $X_{T}^{s s}\left(\mathcal{L}_{\chi}\right)$ and $X_{T}^{s}\left(\mathcal{L}_{\chi}\right)$ by $X^{s s}$ and $X^{s}$, respectively. If $X^{s s} \neq X^{s}$, then the complement $X^{s s} \backslash X^{s}$ is a nonempty $(G \times T)$ invariant closed subset of $X^{s s}$. Hence, the complement $\left(X^{s s} / / T\right) \backslash\left(X^{s} / / T\right)$ is a 
nonempty $G \times\{1\}$-invariant closed subset of $X^{s s} / / T$. In particular, $\left(X^{s s} / / T\right) \backslash$ $\left(X^{s} / / T\right)$ is a finite union of nonempty $G \times\{1\}$-invariant projective varieties. Therefore, there is a $B \times\{1\}$-fixed point in $\left(X^{s s} / / T\right) \backslash\left(X^{s} / / T\right)$. Let

$$
p \in\left(X^{s s} / / T\right) \backslash\left(X^{s} / / T\right)
$$

be a $B \times\{1\}$-fixed point. Let $Y$ be the closed $\{1\} \times T$-orbit in the fiber $\pi^{-1}(\{p\})$ over $p$ for the GIT quotient map $\pi: X^{s s} \longrightarrow X^{s s} / / T$. Since this map $\pi$ is $G \times\{1\}$ equivariant, we conclude that $\pi^{-1}(\{p\})$ is $B \times\{1\}$-invariant. Hence, for any $b \in B$, the translation $(b, 1) \cdot Y$ lies in $\pi^{-1}(\{p\})$. Since the actions of $B \times\{1\}$ and $\{1\} \times T$ on $X$ commute with each other, we see that $(b, 1) \cdot Y$ is also a closed $\{1\} \times T$ orbit in $\pi^{-1}(\{p\})$. By the uniqueness of the closed $\{1\} \times T$-orbit in $\pi^{-1}(\{p\})$ we conclude that $(b, 1) \cdot Y=Y$. Hence $Y$ is preserved by the action of $B \times\{1\}$. In particular, $Y$ is $U \times\{1\}$-invariant, where $U \subset B$ is the unipotent radical. The action of $U \times\{1\}$ on $Y$ induces a homomorphism from $U$ to $T / S$ of algebraic groups, where $\{1\} \times S$ is the stabilizer in $\{1\} \times T$ of some point $q$ in $Y$. Since there is no nontrivial homomorphism from a unipotent group to a torus, we conclude that $U \times\{1\}$ fixes the point $q$.

By [DP, p. 32, Proposition], for any regular dominant character $\chi$ of $T$ with respect to $B$, the morphism $X \hookrightarrow \mathbb{P}\left(V(\chi) \otimes V(\chi)^{*}\right)$ is a $(G \times G)$-equivariant embedding, where $V(\chi)$ is the irreducible representation of $G$ with highest weight $\chi$, and $V(\chi)^{*}$ is its dual. Hence, the $U \times\{1\}$-fixed point set of $X$ is equal to $X \bigcap \mathbb{P}\left(\mathbb{C}_{\chi} \otimes V(\chi)^{*}\right)$, where $\mathbb{C}_{\chi}$ is the one-dimensional $B$-module associated to the character $\chi$. Therefore, by the above discussion, we have $q \in X \bigcap \mathbb{P}\left(\mathbb{C}_{\chi} \otimes V(\chi)^{*}\right)$.

Further, by [DP, Theorem, p. 30] we have

$$
H^{0}\left(X, \mathcal{L}_{\chi}\right)=\bigoplus_{\nu \leq \chi} V(\nu)^{*} \otimes V(\nu)
$$

where the sum runs over all dominant characters $\nu$ of $T$ satisfying $\nu \leq \chi$. By [DP, p. 29, Corollary] and [DP, p. 30, Theorem], the zero locus of

$$
\bigoplus_{\nu<\chi} V(\nu)^{*} \otimes V(\nu) \subset H^{0}\left(X, \mathcal{L}_{\chi}\right)
$$

in $X$ is the unique closed $(G \times G)$-orbit $Z=G / B \times G / B^{-}$. Hence, by the discussion in the previous paragraph, we have $q \in Z$. This contradicts the choice of the polarization $\mathcal{L}_{\chi}$. Therefore, the proof of (a) is complete.

To prove (b), note that $X \backslash X^{s s}$ is a closed subset of $X$, and

$$
Z \backslash Z^{s s}=\left(X \backslash X^{s s}\right) \cap Z .
$$

Also, by Lemma 3.2, the complement $Z \backslash Z^{s s} \subset Z$ is of codimension at least two. Since we have $Z=\bigcap_{i=1}^{n} D_{i}$, the complement $D_{i} \backslash D_{i}^{s s}$ is of codimension at least two for all $1 \leq i \leq n$. Further, every point in the open subset $G \subset X$ is semistable. Hence, $X \backslash X^{s s}$ is of codimension at least three.

The following lemma will be used in the proof of Corollary 3.5. 
LEMMA 3.4

Let $H$ be a reductive algebraic group acting linearly on a polarized projective variety $V$. Assume that $V^{s s}=V^{s}$, where $V^{s s}$ (resp., $V^{s}$ ) is the set of semistable (resp., stable) points of $V$ for the action of $H$. Then the set of all points in $V^{s s}$ whose stabilizer in $H$ is trivial is actually a Zariski-open subset. (It may be empty.)

Proof

Consider the morphism

$$
f: H \times V^{s s} \longrightarrow V^{s s} \times V^{s s}, \quad(h, v) \longmapsto(h \cdot v, v) .
$$

Since $V^{s s}=V^{s}$, this map $f$ is proper (see [MFK, p. 55, Corollary 2.5]). Hence the image

$$
M:=f\left(H \times V^{s s}\right) \subset V^{s s} \times V^{s s}
$$

is a closed subvariety. Now, let

$$
U^{\prime} \subset V^{s s}
$$

be the locus of points with trivial stabilizer (for the action of $H$ ). Take any

$$
v_{0} \in U^{\prime}
$$

and set $z_{0}:=f\left(\left(1, v_{0}\right)\right)=\left(v_{0}, v_{0}\right)$. Then, $\left(f_{*} \mathcal{O}_{H \times V^{s s}}\right)_{z_{0}}$ is a free $\mathcal{O}_{M, z_{0}}$-module of rank one. Hence by [Mu, p. 152, Souped-up version II of Nakayama's lemma], the locus of points $x \in M$ such that $\left(f_{*} \mathcal{O}_{H \times V^{s s}}\right)_{x}$ is a free $\mathcal{O}_{M, x}$-module of rank at most one is a nonempty Zariski-open subset. Since $\left(f_{*}\left(\mathcal{O}_{H \times V^{s s}}\right)\right)_{z}$ is nonzero for all $z \in M$, the set of all points $x \in M$ such that $\left(f_{*}\left(\mathcal{O}_{H \times V^{s s}}\right)\right)_{x}$ is a free $\mathcal{O}_{M, x^{-}}$ module of rank one is a Zariski-open subset of $M$; this Zariski-open subset of $M$ will be denoted by $U$. Note that

$$
f^{-1}(U)=p_{2}^{-1}\left(U^{\prime}\right)
$$

where $p_{2}: H \times V^{s s} \longrightarrow V^{s s}$ is the second projection. Since $p_{2}$ is flat of finite type over $\mathbb{C}$, it is an open map (see [Ha, p. 266, Exercise 9.1]). Hence $U^{\prime}=p_{2}\left(f^{-1}(U)\right)$ is a Zariski-open subset. This finishes the proof of the lemma.

\section{COROLLARY 3.5}

Let $X=\overline{\operatorname{PSL}(n+1, \mathbb{C})}$ be the wonderful compactification of $\operatorname{PSL}(n+1, \mathbb{C}), n \geq 3$. For the choice of the regular dominant character $\chi$ of $T$ as in Proposition 3.3,

(a) the action of $\{1\} \times T$ on $X_{T}^{s s}\left(\mathcal{L}_{\chi}\right)$ is free,

(b) $X_{T}^{s s}\left(\mathcal{L}_{\chi}\right) / / T$ is a smooth projective embedding of $G / T$, and

(c) the set of unstable points $X \backslash\left(X_{T}^{s s}\left(\mathcal{L}_{\chi}\right)\right)$ is a union of irreducible closed subvarieties of codimension at least three.

Proof

Let $\chi$ be a regular dominant character of $T$ as in Proposition 3.3. As in the proof of Proposition 3.3, let $Z$ denote the unique closed $(G \times G)$-orbit in $X$. Also, let 
$X^{s s}, X^{s}, Z^{s s}$, and $Z^{s}$ be as in the proof of Proposition 3.3. By Proposition 3.3 we have $X^{s s}=X^{s}$. Hence by Lemma 3.4, the locus $V$ of points in $X^{s s}$ with trivial stabilizer (for the action of $\{1\} \times T$ ) is a Zariski-open subset of $X^{s s}$. Therefore, $X^{s s} \backslash V$ is a $G \times\{1\}$-stable closed subvariety of $X^{s s}$. By using the arguments in the proof of Proposition 3.3, we see that the set of $B \times\{1\}$-fixed points in $Z \cap\left(X^{s s} \backslash V\right)$ is nonempty. But on the other hand by the proof of [Ka2, p. 194, Example 3.3] we see that, given any point $z \in Z^{s s}$, its stabilizer subgroup in $\{1\} \times T$ is trivial. This is a contradiction. Hence we conclude that the action of $\{1\} \times T$ on $X^{s s}$ is free. This proves parts (a) and (b).

Part (c) follows immediately from the corresponding statement in Proposition 3.3.

\section{Automorphism group of $\overline{\operatorname{PSL}(n+1, \mathbb{C})_{T}^{s s}}(\mathcal{L}) / / T$}

Let $G=\operatorname{PSL}(n+1, \mathbb{C})$, with $n \geq 3$, and define

$$
Y:=\overline{\operatorname{PSL}(n+1, \mathbb{C})_{T}^{s s}}\left(\mathcal{L}_{\chi}\right) / / T,
$$

where $\chi$ is as in Proposition 3.3.

\section{THEOREM 4.1}

Let $A$ denote the connected component, containing the identity element, of the group of holomorphic (i.e., algebraic) automorphisms of $Y$. Then

(a) $A$ is isomorphic to $G$, and

(b) the Picard group of $Y$ is a free abelian group of rank $2 n$.

\section{Proof}

Let $T Y$ denote the algebraic tangent bundle of $Y$. From [MO, Theorem 3.7] we know that $A$ is an algebraic group. The Lie algebra of $A$ is $H^{0}(Y, T Y)$ equipped with the Lie bracket operation of vector fields.

The Lie algebra of $G$ will be denoted by $\mathfrak{g}$. Define $X:=\overline{\operatorname{PSL}(n+1, \mathbb{C})}$, and define $U:=\overline{\operatorname{PSL}(n+1, \mathbb{C})_{T}^{s s}}\left(\mathcal{L}_{\chi}\right)$. The connected component, containing the identity element, of the automorphism group of $X$ is $G \times G$ (see [Br, Example 2.4.5]). From this and the fact that the complement $X \backslash U \subset X$ is of codimension at least three (see Corollary 3.5), we conclude that

$$
H^{0}(U, T U)=H^{0}(X, T X)=\mathfrak{g} \oplus \mathfrak{g} .
$$

Let $\phi: U \longrightarrow Y$ be the geometric invariant theoretic quotient map. Let

$$
T_{U} \supset T_{\phi} \longrightarrow U
$$

be the relative tangent bundle for $\phi$. Since $\phi$ makes $U$ a principal $T$-bundle over $Y$ (see Corollary 3.5(a)), we have the following short exact sequence of vector bundles on $U$ :

$$
0 \longrightarrow T_{\phi} \longrightarrow T U \longrightarrow \phi^{*}(T Y) \longrightarrow 0
$$


and the relative tangent bundle $T_{\phi}$ is identified with the trivial vector bundle $\mathcal{O}_{U} \otimes_{\mathbb{C}} \mathfrak{h}$, where $\mathfrak{h}$ is the Lie algebra of $T$.

Set $Z=X \backslash U$. Since $\operatorname{codim}(Z) \geq 3$ (see Corollary 3.5), we have

$$
H^{0}\left(U, T_{\phi}\right)=H^{0}\left(X, \mathcal{O}_{X} \otimes \mathfrak{h}\right)=\mathfrak{h} .
$$

Note that $H^{1}\left(U, T_{\phi}\right)=H_{Z}^{2}\left(X, \mathcal{O}_{X} \otimes \mathfrak{h}\right)$. Indeed, this follows from the following cohomology exact sequence (see [Gr, Corollary 1.9])

$$
H^{1}\left(X, \mathcal{O}_{X} \otimes \mathfrak{h}\right) \longrightarrow H^{1}\left(U, \mathcal{O}_{X} \otimes \mathfrak{h}\right) \longrightarrow H_{Z}^{2}\left(X, \mathcal{O}_{X} \otimes \mathfrak{h}\right) \longrightarrow H^{2}\left(X, \mathcal{O}_{X} \otimes \mathfrak{h}\right),
$$

combined with the fact that $H^{i}\left(X, \mathcal{O}_{X}\right)=0$ for all $i>0$ (see [DP, p. 30, Theorem]). As $X$ is smooth and $\operatorname{codim}(Z) \geq 3$, it follows from [Gr, Theorem 3.8 and Proposition 1.4] that

$$
H_{Z}^{2}\left(X, \mathcal{O}_{X}\right)=0,
$$

and hence $H^{1}\left(U, T_{\phi}\right)=0$. Now, using this fact in the long exact sequence of cohomologies corresponding to the short exact sequence in (4.1), we obtain the following short exact sequence:

$$
0 \longrightarrow 0 \oplus \mathfrak{h} \longrightarrow \mathfrak{g} \oplus \mathfrak{g} \longrightarrow H^{0}\left(U, \phi^{*} T Y\right) \longrightarrow 0 .
$$

Hence, we have

$$
H^{0}\left(U, \phi^{*} T Y\right)=\mathfrak{g} \oplus(\mathfrak{g} / \mathfrak{h}) .
$$

By using geometric invariant theory, $H^{0}(Y, T Y)$ is the invariant part

$$
H^{0}(Y, T Y)=H^{0}\left(U, \phi^{*} T Y\right)^{\{1\} \times T} \subset H^{0}\left(U, \phi^{*} T Y\right) .
$$

Thus we have $H^{0}(Y, T Y)=\mathfrak{g}$. This proves (a).

To prove (b), let $\left\{D_{i} \mid 1 \leq i \leq n\right\}$ be the $(G \times G)$-stable irreducible closed subvarieties of $\bar{G}$ of codimension one such that

$$
G=\bar{G} \backslash\left(\bigcup_{i=1}^{n} D_{i}\right) .
$$

Let $D_{i}^{s s}=D_{i} \bigcap X^{s s} \subset D_{i}$ be the semistable locus of $D_{i}$. Set $Z:=Y \backslash(G / T)$, and write it as a union

$$
Z=\bigcup_{i=1}^{n} Z_{i},
$$

where each $Z_{i}=D_{i}^{s s} / / T$ is an irreducible closed subvariety of $Y$ of codimension one. As $Y$ is smooth, each $Z_{i}$ produces a line bundle $L_{i} \longrightarrow Y$ whose pullback to $X^{s s}$ is $\mathcal{O}_{X^{s s}}\left(D_{i}^{s s}\right)$. Since $\operatorname{Pic}\left(X^{s s}\right)=\operatorname{Pic}(X)$ and $\left\{\mathcal{O}_{X}\left(D_{i}\right)\right\}_{1 \leq i \leq n}$ are linearly independent in $\operatorname{Pic}(X)$ (see [DP, p. 26, Section 8.1]), we get that $L_{i}, 1 \leq i \leq n$, are linearly independent in $\operatorname{Pic}(Y)$. The Picard group of $G / T$ is isomorphic to the group of characters of the inverse image $\widehat{T}$ of $T$ inside the simply connected covering $\widehat{G}$ of $G$ (see $[\mathrm{KKV}]$ ). Now it follows from the exact sequence in [Fu, Proposition 1.8] that $\operatorname{Pic}(Y)$ is a free abelian group of rank $2 n$, thus completing the proof of (b). 


\section{REMARK 4.2}

The compactification $Y$ of $G / T$ constructed here is an example of a nonspherical variety for the action of $G$ whose connected component of the automorphism group is $G$.

\section{REMARK 4.3}

Note that both $Y$ and $G / B \times G / B$ are smooth compactifications of $G / T$ with isomorphic Picard groups. Further, both are Fano varieties, that is, the anticanonical line bundle is ample. The fact that $G / B \times G / B$ is Fano is well known. That the variety $Y$ is Fano follows as a consequence of the exact sequence in (4.1) together with the facts that $X$ is Fano (see [DP]) and that the codimension of $X \backslash U$ is greater than or equal to three, where $X$ and $U$ are as in the proof of Theorem 4.1. But $Y$ and $G / B \times G / B$ are not isomorphic, as $\operatorname{Aut}^{0}(Y) \simeq G$ and $\operatorname{Aut}^{0}(G / B \times G / B) \simeq G \times G$, where $\operatorname{Aut}^{0}(M)$ denotes the connected component of the group of algebraic automorphisms of a smooth projective variety $M$ containing the identity element.

\section{REMARK 4.4}

Strickland [St] extended the construction of $\bar{G}$ to any arbitrary algebraically closed field. Also, $\bar{G}$ is a Frobenius split variety in positive characteristic (see [St, p. 169, Theorem 3.1]; see [MR] for the definition of Frobenius splitting). Since $T$ is linearly reductive, using the Reynolds operator, one can see that the geometric invariant theoretic quotient of $\bar{G}$ for the action of $T$ is also Frobenius split for any polarization on $\bar{G}$.

Acknowledgments. We are grateful to the referee for comments to improve the exposition. The first author thanks the Institute of Mathematical Sciences for hospitality while this work was carried out.

\section{References}

[Br] M. Brion, The total coordinate ring of a wonderful variety, J. Algebra 313 (2007), 61-99. MR 2326138. DOI 10.1016/j.jalgebra.2006.12.022.

[DP] C. De Concini and C. Procesi, "Complete symmetric varieties" in Invariant Theory (Montecatini, 1982), Lecture Notes in Math. 996, Springer, Berlin, 1983, 1-44. MR 0718125. DOI 10.1007/BFb0063234.

[Fu W. Fulton, Intersection Theory, 2nd ed., Ergeb. Math. Grenzgeb. (3) 2, Springer, Berlin, 1998. MR 1644323. DOI 10.1007/978-1-4612-1700-8.

[Gr] A. Grothendieck, Local Cohomology, Lecture Notes in Math. 41, Springer, Berlin, 1967. MR 0224620.

[Ha] R. Hartshorne, Algebraic Geometry, Grad. Texts in Math. 52, Springer, Berlin, 1977. MR 0463157. 
[Hu1] J. E. Humphreys, Introduction to Lie Algebras and Representation Theory, Grad. Texts in Math. 9, Springer, Berlin, 1972. MR 0323842.

[Hu2] Linear Algebraic Groups, Grad. Texts in Math. 21, Springer, Berlin, 1975. MR 0396773.

[Ka1] S. S. Kannan, Torus quotients of homogeneous spaces, II, Proc. Indian Acad. Sci. Math. Sci. 109 (1999), 23-39. MR 1687020. DOI 10.1007/BF02837764.

[Ka2] _ "GIT related problems of the flag variety for the action of a maximal torus" in Groups of Exceptional Type, Coxeter Groups and Related Geometries, Springer Proc. Math. Stat. 82, Springer, New Delhi, 2014, 189-203. MR 3207277. DOI 10.1007/978-81-322-1814-2_10.

[KKV] F. Knop, H. Kraft, and T. Vust, "The Picard group of a $G$-variety" in Algebraische Transformationsgruppen und Invariantentheorie, DMV Sem. 13, Birkhäuser, Basel, 1989, 77-87. MR 1044586.

[MO] H. Matsumura and F. Oort, Representability of group functors, and automorphisms of algebraic schemes, Invent. Math. 4 (1967), 1-25. MR 0217090.

[MR] V. B. Mehta and A. Ramanathan, Frobenius splitting and cohomology vanishing for Schubert varieties, Ann. of Math. (2) 122 (1985), 27-40. MR 0799251. DOI 10.2307/1971368.

[Mu] D. Mumford, The Red Book of Varieties and Schemes, 2nd expanded ed., Lecture Notes in Math. 1358, Springer, Berlin, 1999. MR 1748380. DOI $10.1007 /$ b62130.

[MFK] D. Mumford, J. Fogarty, and F. Kirwan, Geometric Invariant Theory, 3rd ed., Ergeb. Math. Grenzgeb. (3) 34, Springer, Berlin, 1994. MR 1304906. DOI 10.1007/978-3-642-57916-5.

[Se] C. S. Seshadri, Quotient spaces modulo reductive algebraic groups, Ann. of Math. (2) 95 (1972), 511-556. MR 0309940.

[St] E. Strickland, A vanishing theorem for group compactifications, Math. Ann. 277 (1987), 165-171. MR 0884653. DOI 10.1007/BF01457285.

Biswas: School of Mathematics, Tata Institute of Fundamental Research, Mumbai, India; indranil@math.tifr.res.in

Kannan: Chennai Mathematical Institute, Siruseri, Kelambakkam, India;

kannan@cmi.ac.in

Nagaraj: Institute of Mathematical Sciences, Central Institutes of Technology

Campus, Taramani, Chennai, India; dsn@imsc.res.in 